\title{
A MicroRNA Signature in Gestational Diabetes Mellitus Associated with Risk of Macrosomia
}

\author{
Jiandong $\mathrm{Li}^{\mathrm{a}}$ Liping Song ${ }^{\mathrm{d}}$ Ling Zhou ${ }^{\mathrm{e}}$ Jiaoxiang Wu Chunjie Sheng ${ }^{\mathrm{a}}$ \\ Huiming Chen ${ }^{\mathrm{a}}$ Yanjun Liu ${ }^{\mathrm{b}}$ Shijuan Gao ${ }^{\mathrm{a}}$ Wenlin Huanga,c \\ ${ }^{a}$ CAS Key Laboratory of Pathogenic Microbiology and Immunology, Institute of Microbiology, Chinese \\ Academy of Sciences, Beijing, ${ }^{\text {bDepartment }}$ of Endocrinology, the $306^{\text {th }}$ Hospital of P.L.A, Beijing, ${ }^{\text {CThe }}$ \\ Key Laboratory of Tumor Targeted Medicine in Guangdong Province, Guangzhou Doublle Bio-product \\ Inc., Guangzhou, 'Beijing Municipal Center for Food Safety Monitoring and Risk Assessment, Beijing, \\ eDepartment of Obstetrics and Gynecology, the $306^{\text {th }}$ Hospital of P.L.A, Beijing, China
}

\section{Key Words}

Gestational diabetes mellitus • Cell signaling • MicroRNAs • EGFR

\begin{abstract}
Background/Aims: MicroRNA (miRNA) is a small non-coding RNA molecule that functions in regulation of gene expression by targeting mRNA to affect its stability and/or translation. The aim of this study was to evaluate the miRNAs involvement in gestational diabetes mellitus (GDM), a well known risk factor for fetal overgrowth. Methods: Differential microRNA expression in placental tissues of normal controls and women with GDM were identified by miRNA micorarray analysis and further confirmed by quantitative real-time PCR (qRT-PCR) on an independent set of normal and GDM placental tissues. Target genes of microRNAs were bioinformatically predicted and verified in vitro by Western blotting. Results: Our results uncovered 9 miRNAs that were significantly deregulated in GDM samples: miR-508-3p was up-regulated and miR-27a, miR-9, miR-137, miR-92a, miR-33a, miR-30d, miR-362-5p and miR-502-5p were down-regulated. Bioinformatic approaches revealed that the microRNAs signature identifies gene targets involved in EGFR (epidermal growth factor receptor)-PI3K (phosphoinositide 3-Kinase)-Akt (also known as protein kinase B) pathway, a signal cascade which plays important roles in placental development and fetal growth. We found that the protein levels of EGFR, PI3K and phospho-Akt were up-regulated and PIKfyve (a FYVE fingercontaining phosphoinositide kinase), a negative regulator of EGFR signaling, was downregulated significantly in GDM tissues. We also confirmed PIKfyve was a direct target of miR508-3p. Conclusion: Our data identified a miRNA signature involvement in GDM which may contribute to macrosomia through enhancing EGFR signaling.
\end{abstract}

Wenlin Huang, Shijuan Gao and Yanjun Liu

\section{KARGER 125}

CAS Key Laboratory of Pathogenic Microbiology and Immunology, Institute of Microbiology, Chinese Academy of Sciences, Beijing 100101, (PR China) or Department of Endocrinology, the $306^{\text {th }}$ Hospital of P.L.A, Beijing 100101, (China)

E-Mail hwenl@mail.sysu.edu.cn, E-Mail gaoshj@im.ac.cn and

E-Mail damaoermaosanmao@yahoo.com.cn 


\section{Cellular Physiology Cell Physiol Biochem 2015;37:243-252

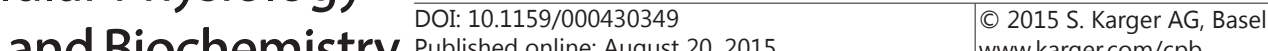 and Biochemistry Published online: August 20, $2015 \quad$ www.karger.com/cpb \\ Li et al.: A MicroRNA Signature in Gestational Diabetes Mellitus}

\section{Introduction}

Gestational diabetes mellitus (GDM) is defined as any degree of glucose intolerance that is first detected during pregnancy. GDM is a risk factor for pregnancy-related maternal and fetal morbidity. Babies born to mothers with GDM are typically at increased risk of excessive birth weight or macrosomia. A macrosomic foetus is defined as weighing more than $4 \mathrm{~kg}$. Moreover, macrosomic babies are prone to developing childhood obesity and type 2 diabetes and/or cardiovascular diseases in the later stage of life [1-3].

Growth factors within the maternal circulation, such as insulin-like growth factors 1 and 2 (IGF1 and IGF2), epidermal growth factor (EGF) and platelet-derived growth factor (PDGF) have been shown to be important regulators of placental development and fetal growth [2]. Because these growth factors exert their effects via intracellular signaling cascades, disrupted or dysregulated growth factor signaling pathways are linked to aberrant fetal growth. The roles of EGF/EGFR signaling cascade for placental development and fetal growth have been confirmed in vitro and in vivo. Alternations of EGFR function are associated with reduced embryonic growth in humans [4]. EGFR-deficient mice had significant smaller placentas and displayed severe fetal growth restriction [5]. The results from in vitro study using human placental cell lines indicated that EGF promotes trophoblast proliferation and inhibits trophoblast apoptosis [6]. However, the detailed mechanisms by which EGFR signaling cascade is deregulated in placenta are not well known.

MicroRNA (miRNA), a small non-coding, single-stranded RNA of 20 22 nucleotides long, is recognized as one of key post-transcriptional regulators of gene expression by binding to the 3' untranslated region (UTR) of specific genes to affect mRNAs stability and/ or translation. MiRNA is involved in a variety of cellular processes, for example, proliferation, differentiation, apoptosis and development. Aberrant expression of miRNA has been detected in many diseases, such as cancer, type 2 diabetes as well as cardiovascular neurological and autoimmune diseases [7-10]. Previous studies have suggested that miRNAs are involved in GDM [11]. However, the miRNA expression profiles in placenta tissues from GDM patients and its biological significance, to our knowledge, have not been evaluated.

In the present study, we compared the miRNA expression profiles of placenta samples from healthy and GDM women by microarray analysis. Nine miRNAs have been further confirmed to be significantly deregulated in GDM samples by qRT-PCR analysis. These deregulated miRNAs identify gene targets involved in EGFR/PI3K/Akt signaling cascade which plays essential roles in regulating fetal growth during pregnancy $[2,12]$. Our data identify a specific miRNA signature in GDM and provide new potential insights into the mechanisms of enhanced fetal growth in GDM patients.

\section{Materials and Methods}

\section{Patients}

The subjects were recruited in the Department of Obstetrics and Gynecology, the $306^{\text {th }}$ Hospital of P.L.A, Beijing, China. This study was approved by an institutional review board, and placental tissue samples were collected after informed consent. The pregnant women were 21 to 37 years old. Placentas were obtained from 15 healthy women and 15 women with GDM immediately after delivery, frozen and kept in liquid nitrogen until RNA or protein isolation. Delivered newborns were immediately weighed after delivery. The mean birth weight of babies from GDM mothers (4042 $\pm 104 \mathrm{~g}$ ) was higher than babies from healthy mothers $(3368 \pm 69 \mathrm{~g})(\mathrm{P}<0.001)$. Selected control women had no significant history of illness, no pregnancy-related complications. GDM was diagnosed when fasting blood glucose $>5.1 \mathrm{mmol} / \mathrm{L}$ according to the Endocrine Society criteria.

\section{Microarray miRNA expression profiling and data analysis}

Total RNA was isolated from equal amounts of placental tissue collected from normal controls and GDM patients using RecoverAllTM Total Nucleic Acid Isolation kit (Ambion) according to the manufacturer's protocol. The purities and concentrations of RNA were determined by NanoDrop ND-2000 (Thermo). 


\section{Cellular Physiology Cell Physiol Biochem 2015;37:243-252 \begin{tabular}{l|l|l} 
DOI: 10.1159/000430349 & C 2015 S. Karger AG, Basel
\end{tabular} and Biochemistry Published online: August 20, 2015

MicroRNA profiling of 10 placental samples ( 5 from normal controls and 5 from GDM patients) were assessed by using Agilent Human miRNA Microarray (Agilent) according to the manufacture's protocol. The chip was scanned by Agilent Microarray Scanner and images were analyzed by Feature Extraction software 10.7 (Agilent). Data were normalized by quantile algorithm, Gene Spring Software 11.0 (Agilent). Differentially expressed miRNAs were selected with $\mathrm{p}<0.05$.

\section{miRNA targets predication}

TargetScan (http://www.targetscan.org/) and miRanda (http://www.microrna.org/microrna) were used to predict putative miRNA targets. To minimize the number of false predication, we performed the union and intersection operator in Excel to obtain the common targets.

$q R T$ - PCR

To validate the expression of deregulated miRNAs in an independent set of normal $(n=10)$ and GDM $(\mathrm{n}=10)$ placental tissues, total RNA was extracted with TRNzol reagent (TIANGEN Biotech, Beijing, China) and reverse transcribed into cDNAs with miRNA first-strand cDNA kit (TIANGEN Biotech, Beijing, China). Quantitative real-time PCR was performed by using miRcute miRNA qPCR detection kit (TIANGEN Biotech, Beijing, China) following manufacturer's instruction. PCR reactions were conducted in 7300 Real-Time PCR System (Applied Biosystems, Foster City, CA). U6 snRNA was used as internal control for normalization. Relative expression for miRNA was determined using $2^{-\Delta \Delta C t}$ method. All reactions were performed in triplicate.

For mRNA analysis, cDNA was synthesized from total RNA using RevertAid First Strand cDNA Synthesis kit (Thermo Scientific, Waltham, MA) and quantitative real-time PCR was performed by using SYBR Green qPCR Master Mix (Thermo Scientific, Waltham, MA). The cycle conditions included an initial denaturation step at $95^{\circ} \mathrm{C}$ for 30 seconds followed by 40 cycles of amplification for 3 seconds at $95^{\circ} \mathrm{C}$ and 1 minute at $60^{\circ} \mathrm{C}$. The housekeeping gene GAPDH was used as an internal control. The sequences of gene specific primers used for qRT-PCR were as follows: EGFR (forward: 5'-GTT TGG GAG TTG ATG ACCTT-3'; reverse: 5'-GGA ACT TTG GGC GAC TATCT-3'), PIK3CA (forward: 5'-TGG ATG CTC TAC AGG GCTTT-3'; reverse: 5'-GTC TGG GTT CTC CCA ATTCA-3'), PIK3CB (forward: 5'-GCA TTA AAA GGG AGC GAGTG-3'; reverse: 5'-CAT GCC GTC GTA AAA TCAGA-3'), PIK3CD (forward: 5'-CTG GCT GAA GTC CAA GAACC-3'; reverse: 5'-CTC GGA TCA TGA TGT TGTCG-3'), PIK3CG (forward: 5'-ATA CCA TGA TAG CGC CCTTG-3'; reverse: 5'-AAT CACA GCG AAC CTC TGCT-3'), PIK3R2 (forward: 5'-AGA TGG GCA CTA TGG CTT CTCAG-3'; reverse: 5'-CCT CCA CGC TGT CCT CCTTG-3'), PIK3R3 (forward: 5'-GGA GGA GGT AAA TGA CAAAT-3'; reverse: 5'-CCT TCC GCA AAG TCA AAGTA-3'), Akt (forward: 5'-CTG GGC AAG GGC ACT TTCGG-3'; reverse: 5'-AGG CGG TCG TGG GTC TGGAA-3'), PIKfyve (forward: 5'-TGT AAA GAA TAC CGA AATGC-3'; reverse: 5'-TAA TCT GAT AGG GCT GCTAC-3'), GAPDH (forward: 5'-GGA GAA ACC TGC CAA GTATG-3'; reverse: 5'-TTA CTC CTT GGA GGC CAT GTAG-3').

\section{Western blotting}

Total proteins were extracted from cells or tissues by using cell lysis buffer (Cell Signaling Technology, Danvers, MA) and separated by SDS-PAGE and transferred to nitrocellulose membrane (Amersham Biosciences, Amersham, UK). After blocking, the membrane was incubated with primary antibodies overnight at $4^{\circ} \mathrm{C}$ followed by washing and incubation with a horseradish peroxidase-conjugated secondary antibody for 2 hours at room temperature. Bands were detected using enhanced chemiluminescence (Applygen, Beijing, China). Relative band intensity was evaluated by Image J software (NIH). Primary antibodies used in this study were as follows: rabbit anti-EGFR (\#2232; CST), anti-p110- $\gamma$ (\#4252; CST), anti-p85- $\beta$ (ab137815; Abcam), anti-Akt (Ab-308, 21055; Signalway Antibody, MD), anti-p-Akt (Ser 473, 11054; Signalway Antibody, MD), mouse anti-PIKfyve (sc-100408; Santa Cruz) and anti- $\beta$-actin (6a3; Sungene Biotech, China) antibodies.

\section{Cell lines and transfection}

HTR-8/SVneo human trophoblast cells were cultured in RPMI 1640 (HyClone, South Logan, USA) supplemented with $10 \%$ heat-inactivated fetal bovine serum (Gibco, Paisley, United Kingdom) at $37^{\circ} \mathrm{C}$ in a humidified 5\% $\mathrm{CO}_{2}$ incubator. HTR-8/SVneo cells were transfected with plasmids or miR-508-3p mimics, miR-508-3p inhibitor, or miRNA negative control (RiboBio, Guangzhou, China) using Lipofectamine 2000 (Invitrogen, Carsland, CA) according to manufacturer's instruction. 


\section{Cellular Physiology Cell Physiol Biochem 2015;37:243-252 \begin{tabular}{l|l|l}
\hline DOI: $10.1159 / 000430349$ & C 2015 S. Karger AG, Basel
\end{tabular} www.karger.com/cpb

\section{Dual-luciferase reporter assay}

TodeterminewhethermiR-508-3ptargetsPIKfyve,a274-bpfragmentofPIKfyve3'UTRcontainingputative binding site of miR-508-3p was amplified by PCR with primers: 5'-GCTCTAGACACAGGTGCAGTGTATTA-3' (forward) and 5'-GCTCTAGAGTTCAAGGCAGTTATCTT-3' (reverse). The amplified products were inserted into the XbaI site of pRL-TK vector (Promega, Madison, WI). The mutated pRL-TK-PIKfyve-3'UTR construct was generated with Site-Directed Mutagenesis Kit (SBS Genetech, Beijing, China). HTR-8/SVneo cells were transfected with pRL-TK-PIKfyve-3'UTR, pGL3-control along with miR negative control (NC), miR-508-3p mimics, or miR-508-3p inhibitor for $36 \mathrm{~h}$. The cells were harvested, and a luciferase assay was performed using Dual-Luciferase Assay System (Promega, Madison, WI) according to manufacturer's instructions.

\section{Statistical analysis}

Data are presented as mean \pm standard deviation (S.D.) of three independent experiments. Statistical comparisons were performed using SPSS 11.5. Student's $t$ test was used to evaluate individual differences between groups and analysis of variance (ANOVA) was used for multiple comparisons. Values of $p<0.05$ are considered to be statistically significant.

\section{Results}

Identification of microRNAs differentially expressed in placental tissues of GDM patients

To investigate miRNAs involvement in GDM, we applied a high-throughput human miRNA array analysis with placental tissues from 5 normal controls and 5 GDM patients. Comparative analysis of the miRNA expression arrays between normal and GDM samples identified 29 miRNAs with significant differences $(\mathrm{p}<0.05)$ including upregulation of 3 miRNAs and downregulation of 26 miRNAs (Fig. 1).

The microRNAs signature identifies gene targets involved in EGFR/PI3K/Akt pathway

To investigate the biological roles of the deregulated miRNAs in GDM, we predicted the gene targets for those 29 identified miRNAs by using the programs TargetScan, (http:// www.targetscan.org/) and miRanda (http://www.microrna.org/microrna). Interestingly, we obtained 15 miRNAs that target genes involvement in EGFR/PI3K/Akt pathway (Fig. 2A). To validate this miRNA signature, we performed qRT-PCR confirmation experiments on an independent set of placental tissues from normal controls $(n=10)$ and GDM patients $(n=10)$. QRT-PCR analysis showed a statistically significant deregulation of 9 miRNAs with miR-508-

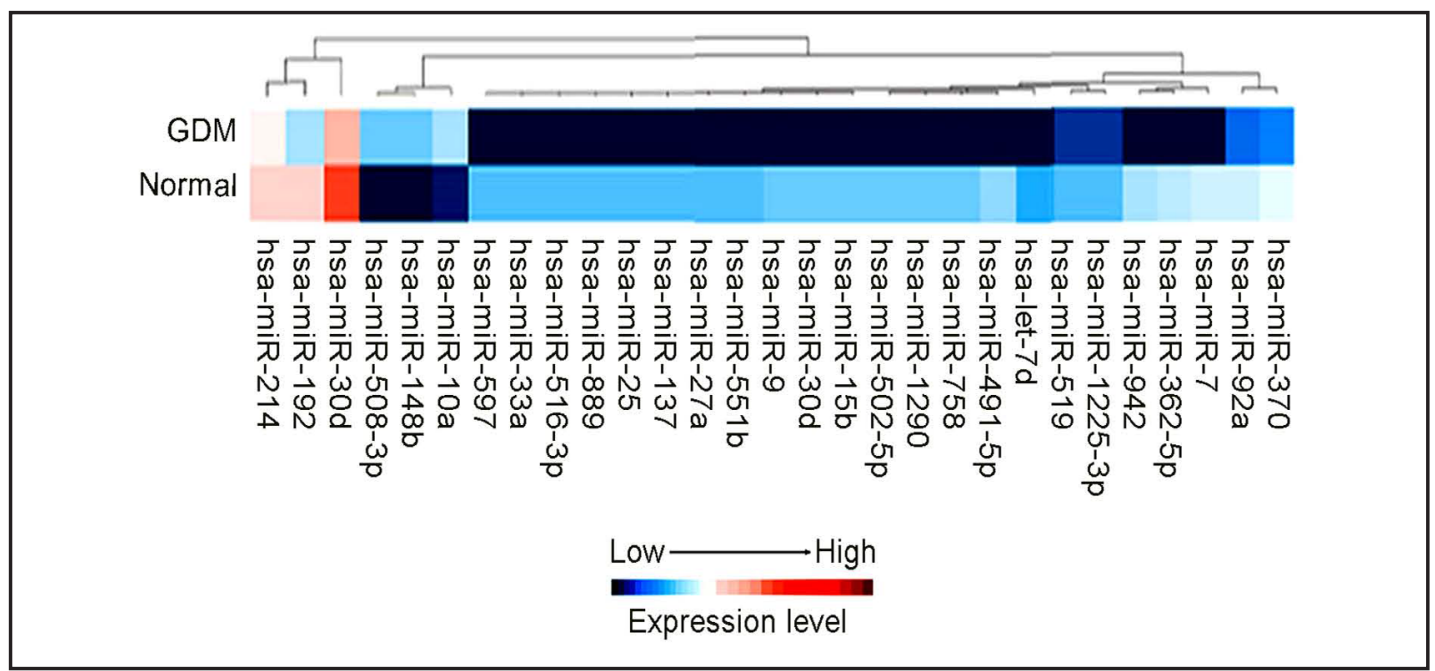

Fig. 1. Deregulated miRNAs in placental tissues of normal controls vs. women with GDM. miRNA expression profiling of terminal placental tissues from GDM $(n=5)$ and normal control $(n=5) .29$ significant deregulated miRNAs in GDM patients were identified. 
A

\begin{tabular}{|c|c|c|}
\hline & miRNA & Predicted target genes \\
\hline \multirow{12}{*}{ 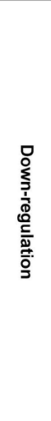 } & $m i R-370$ & FoxO1 \\
\hline & miR-92a & PIK3R3 \\
\hline & $\operatorname{miR}-7$ & PIK3R3, PIK3CD, EGFR \\
\hline & miR-362-5p & Akt \\
\hline & miR-502-5p & PIKЗCG \\
\hline & $\operatorname{miR}-15 b$ & PIK3R1,Akt \\
\hline & miR-9 & PIK3R3 \\
\hline & $\operatorname{miR}-27 a$ & PIK3CD, EGFR \\
\hline & $\operatorname{miR}-137$ & PIK3R3, Akt \\
\hline & $\operatorname{miR}-25$ & PIK3R3, PIKfyve \\
\hline & miR-33a & PIK3R3 \\
\hline & miR-30d & PIK3CD, PIK3R2 \\
\hline \multirow{3}{*}{ 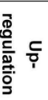 } & miR-508-3p & PIKfyve \\
\hline & miR-148b & PIK3R3 \\
\hline & miR-10a & PIK $3 C A$ \\
\hline
\end{tabular}

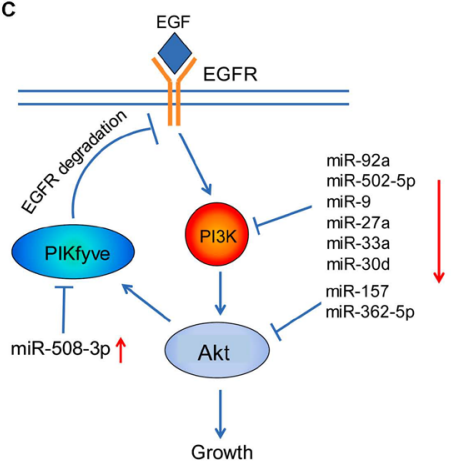

B
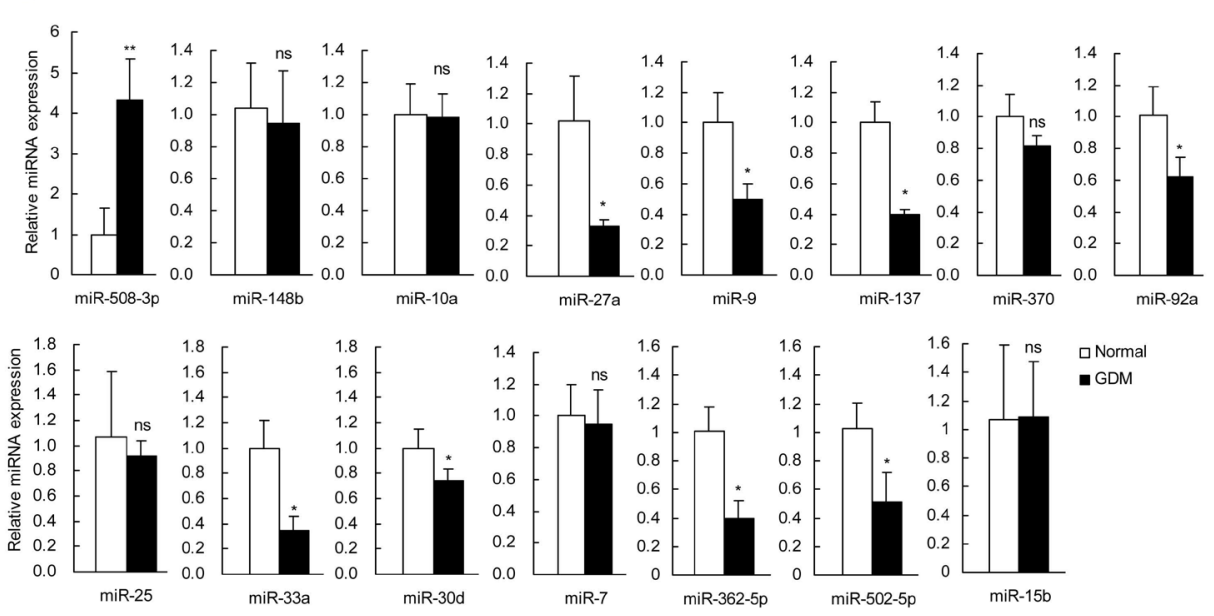

Fig. 2. The microRNAs signature identifies gene targets involved in EGFR/PI3K/Akt pathway. (A) Target genes for those 29 identified miRNAs were predicted by TargetScan. Candidate target genes of 15 deregulated miRNAs coupled to EGFR/PI3K/Akt pathway. Red miRNA indicated that which has been confirmed to be deregulated significantly in placental tissues of GDM patients on an independents set of normal and GDM samples as performed in (B). (B) Quantitative real-time PCR analysis of relative expressions of the 15 miRNAs in an independent set of normal $(n=10)$ and GDM $(n=10)$ placental tissues. Data represent mean \pm S.D. of three independent experiments. Statistical significance was determined by Student's $t$-test. *, $p<0.05$; $* *, p<0.01$; n.s.: no significant difference. (C) Diagram illustrating a proposed model for how deregulated miRNAs in women with GDM interfere with fetal growth through EGFR/PI3K/Akt signaling pathway. EGF and EGFR increase during pregnancy. EGFR regulates fetal development and growth by activating the PI3K/ Akt pathway. Akt activation phosphorylates and activates the kinase PIKfyve which promotes EGFR degradation. Thus, Akt phosphorylation and activation of PIKfyve is a feedback mechanism for terminating EGFR signaling. Downregulation of the miRNAs that target PI3k/Akt and upregulation of the miRNA targeting PIKfyve will enhance EGFR signaling and prevent termination of its signaling.

3p up-regulation and miR-27a, miR-9, miR-137, miR-92a, miR-33a, miR-30d, miR-362-5p and miR-502-5p downregulation. Six miRNAs (miR-148b, miR-10a, miR-370, miR-25 and miR-15b) were found to be non-significant in the two cohorts of placental tissues (normal vs. GDM) (Fig. 2B).

EGFR/PI3K/Akt pathway plays important roles in placental development and fetal growth, and we identified that the mean birth weight of babies from GDM mothers (4042 \pm 


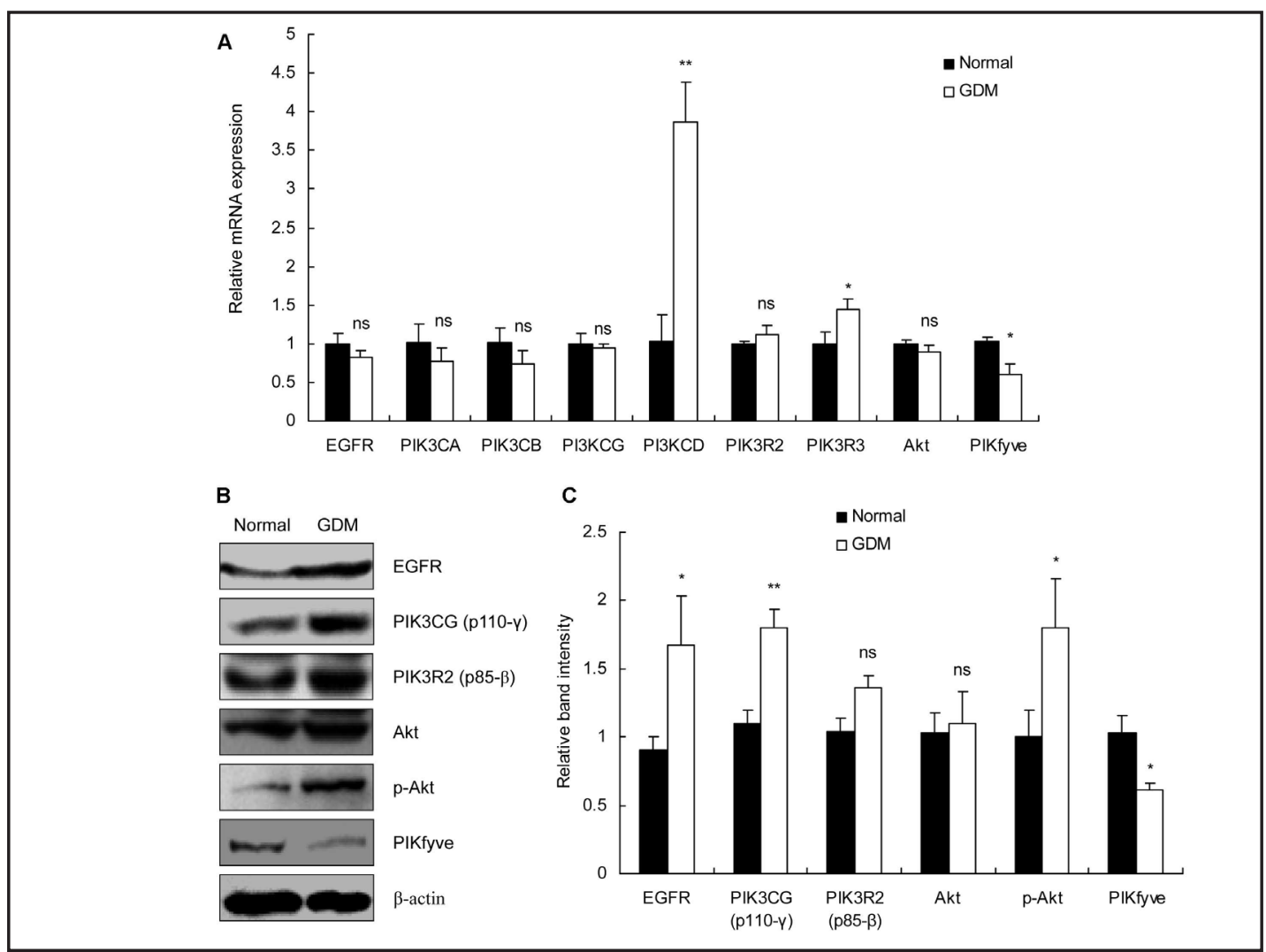

Fig. 3. Expression of EGFR/PI3K/Akt pathway-related genes in normal vs. GDM placental tissues. Total RNAs or proteins were extracted form terminal placental tissues of normal controls and women with GDM. mRNA levels of the indicated genes were evaluated by quantitative RT-PCR with GAPDH as an internal control (A) and protein levels were analysed by Western blots with the indicated antibodies. Relative protein band intensities were measured using Image J software (NIH). Data represent mean \pm S.D. of at least three independent experiments. Statistical significance was determined by Student's $t$-test. ${ }^{* *} P<0.01,{ }^{*} P<0.05$, ns: no significant difference.

$104 \mathrm{~g})$ was higher than babies from healthy mothers $(3368 \pm 69 \mathrm{~g})(\mathrm{P}<0.001)$. As a result, it is conceivable that this 9 miRNA signature may contribute to the regulation of fetal growth through the regulation of EGFR/PI3K/Akt signal cascade. Here we present a proposed model for how deregulated miRNAs in women with GDM interfere with fetal growth through EGFR/PI3K/Akt signaling pathway. EGF and EGFR increase during pregnancy. EGFR regulates fetal development and growth by activating the PI3K/Akt pathway. Akt activation phosphorylates and activates the kinase PIKfyve which promotes EGFR degradation. Thus, Akt phosphorylation and activation of PIKfyve is a feedback mechanism for terminating EGFR signaling and reducing EGFR abundance [13]. Downregulation of the miRNAs that target PI3K/Akt and upregulation of the miRNAs targeting PIKfyve will enhance EGFR signaling and prevent termination of its signaling.

Expressions of EGFR/PI3K/Akt pathway-related genes in normal vs. GDM placental tissues

We then detected the predicted gene targets of the desregulated miRNAs identified here by qRT-PCR and western blot analysis respectively. Total RNAs or proteins were extracted from terminal placental tissues of normal controls $(n=5)$ and women with GDM $(n=5)$ and qRT-PCR analysis was performed. As shown in Fig. 3A, the mRNA levels of PIK3CD (p110- $\delta$ ) and PIK3R3 (p55- $\gamma)$ were up-regulated $(* *, p<0.01 ; *, p<0.05)$ and PIKfyve was downregulated $(*, p<0.05)$ in GDM tissues; while the mRNA levels of EGFR, PIK3CA (p110- $\alpha)$, PIK3CB (p110- $\beta$ ), PIK3CG (p110- $\gamma)$, PIK3R2 (p85- $\beta$ ) and Akt were found to be nonKARGER 


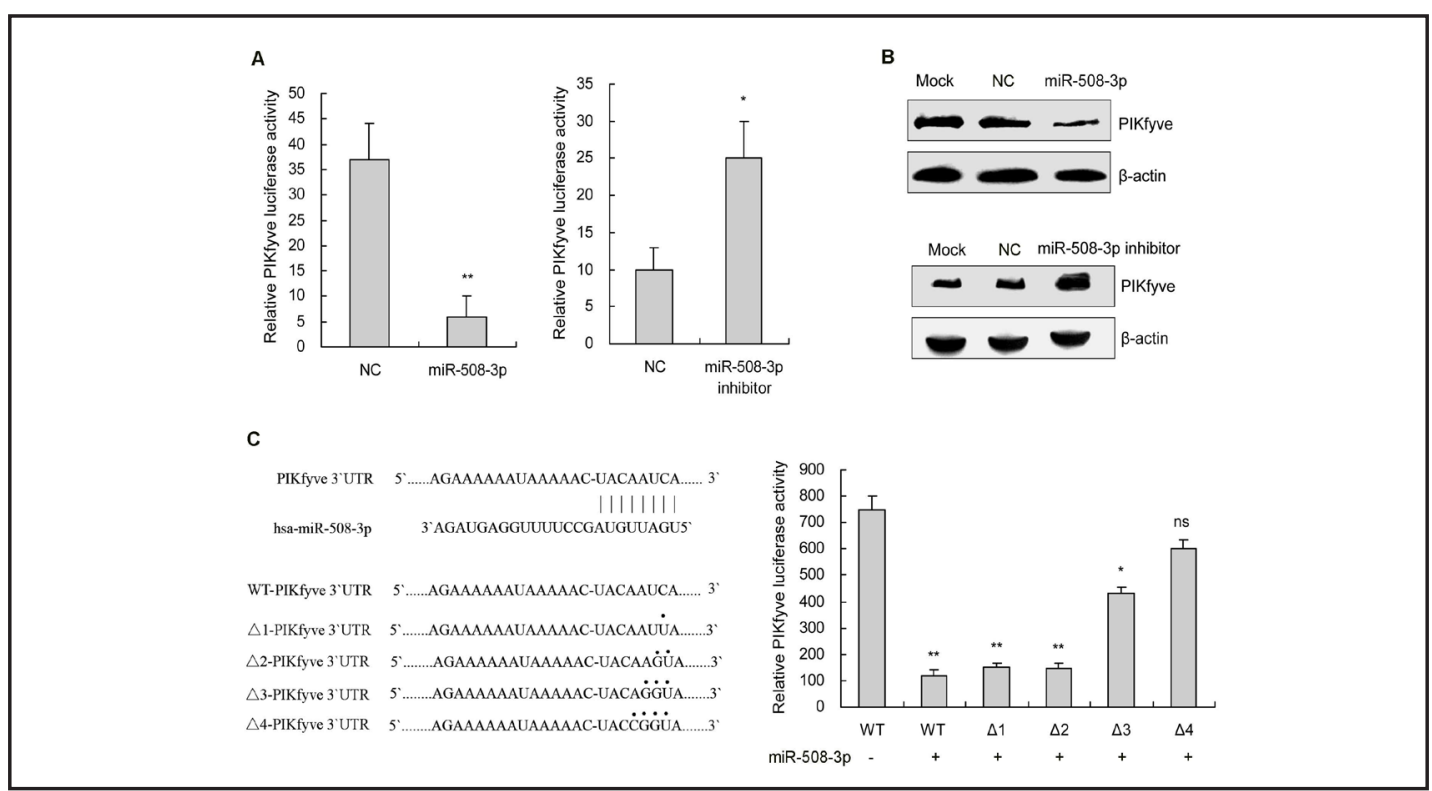

Fig. 4. PIKfyve is a direct target of miR-508-3p. (A) HTR-8 cells were transfected with pRL-TK-PIKfyve 3'UTR, pGL3-control along with miR negative control (NC), miR-508-3p mimics or miR-508-3p inhibitor for $36 \mathrm{~h}$. The cells were harvested, and a luciferase assay was performed. (B) HTR-8 cells were transfected with miR negative control (NC), miR-508-3p mimics or miR-508-3p inhibitor for $36 \mathrm{~h}$. The cells were harvested and PIKfyve protein levels were analyzed by Western blotting. (C) Wild-type or mutated miR-508-3p putative targets on PIKfyve 3'-UTR were cloned into pRL-TK reporter plasmid as indicated. HTR-8 cells were transfected with wild type or mutant pRL-TK- PIKfyve 3'UTR construct as indicated, pGL3-control along with miR-508-3p mimics or miR-NC for $36 \mathrm{~h}$. The cells were harvested, and a luciferase assay was performed. Data are representative of at least three independent experiments. Statistical significance was determined by Student's $t$-test (B) and One-way ANOVA analysis (C). ${ }^{*}, p<0.05$; $^{* *}, p<0.01$; n.s.: no significant difference.

significant in normal and GDM samples. Because miRNAs suppress gene expression either by promoting mRNA degradation or by inhibiting translation, depending on the degree of complementarity with target mRNA sequences, we then assessed the expressions of EGFR/ PI3K/Akt pathway-related genes in protein levels by using western blot assays. As shown in Fig. 3B and 3C, the protein levels of EGFR, PIK3CG (p110- $\gamma$ ) and p-Akt were up-regulated and PIKfyve was down-regulated significantly in GDM tissues $(* *, p<0.01 ; *, p<0.05)$. Of note, the significant increase in the expression of EGFR, p110- $\gamma$ and p-Akt and the decrease in the levels of PIKfyve in GDM tissues suggest that enhanced EGFR signaling exists in GDM tissues which may contribute to enhanced fetal growth.

\section{PIKfyve is a direct target of miR-508-3p}

MiR-508-3p is the most highly expressed miRNA in placental tissues of GDM patients identified here. On the basis of miRNA target gene prediction database (TargetScan, http:// www.targetscan.org/ and miRanda, http://www.microrna.org/microrna), PIKfyve was predicted as a target gene of miR-508-3p. We then confirmed whether miR-508-3p targeted PIKfyve. To this end, we cloned a 274-bp fragment of PIKfyve 3'UTR containing putative binding site of miR-508-3p into the pRL-TK vector immediately downstream of the firefly luciferase gene to construct the pRL-TK-PIKfyve-3'UTR reporter plasmid. HTR-8 cells were transfected with pRL-TK-PIKfyve-3'UTR vector, pGL-3 which used as an internal control, along with miR-508-3p mimics, miR-508-3p inhibitor or their corresponding negative controls. As shown in Fig. 4A, miR-508-3p overexpression inhibited activity of pRL-TKPIKfyve-3'UTR. Conversely, suppression of miR-508-3p expression enhanced pRL-TKPIKfyve-3'UTR activity, suggesting that miR-508-3p regulates directly PIKfyve expression. We also investigated whether miR-508-3p had a regulatory effect on endogenous PIKfyve 


\section{Cellular Physiology Cell Physiol Biochem 2015;37:243-252 \\ \begin{tabular}{c|c|c} 
DOI: 10.1159/000430349 & $\begin{array}{l}\text { O 2015 S. Karger AG, Base } \\
\text { www.karger.com/cpb }\end{array}$ \\
and Biochemistry & Published online: August 20, 2015 &
\end{tabular} \\ Li et al.: A MicroRNA Signature in Gestational Diabetes Mellitus}

expression. Data from Western blotting analysis indicated that miR-508-3p suppressed PIKfyve protein expression while miR-508-3p inhibitor increased PIKfyve protein levels in HTR-8 cells (Fig. 4B). To clarify the specific binding sites, mutated pRL-TK-PIKfyve$3^{\prime}$ UTR vectors with some nucleotides mutation in the putative seed-binding region were constructed and transfected together with miR-508-3p mimics into HTR-8 cells. Compared with wild-type vector, there was no significant influence on the luciferase activity in the cells cotransfected with vector carrying sequences mutated from 5'-AAUC-3' to 5'-CGGU-3' and miR-508-3p mimics (Fig. 4C). Together, these results suggest that miR-508-3p directly targets PIKfyve via its 3'UTR.

\section{Discussion}

GDM is one of the most common pregnancy complications and a well known risk factor for macrosomia [14]. Dysregulated expressions of miRNAs have been shown to be potentially disease-specific and may contribute to pathologic processes [8]. However, no reported study investigates the miRNA signature in placental tissues from GDM. To identify specific miRNA signature and its biological significance in the pathobiology of GDM, we performed miRNA profiling in placental tissues from normal and GDM patients. Our combined data set, which included array-based identification followed by qRT-PCR, uncovered 9 miRNAs with significant deregulation associated with EGFR/PI3K/Akt signaling cascade. Furthermore, by qRT-PCR and western blot analysis, we have demonstrated that enhanced EGFR signaling exists in the GDM samples. Together, our results suggest these deregulated miRNAs identified here might be implicated in GDM and in part in the pathology of fetal overgrowth via EGFR/ PI3K/Akt signaling cascade.

EGF-mediated activation of the PI3K/Akt pathway has been shown to be important in promoting placenta development and fetal growth in both humans and rodents $[2,15]$. EGFR-deficient mice had significantly smaller placentas and displayed severe fetal growth [5].Cord serum EGF concentrations increase in GDM pregnancies [1]. However, EGF has been reported not to cross the placenta barrier [16]. How EGF concentrations are increased and by which the downstream signaling molecules are regulated in placenta is not wellunderstood. Here, based on the observation that 8 down-regulated miRNAs in GDM inversely correlate with EGFR/PI3K/Akt pathway-related genes and one up-regulated miRNA targets the negative regulator of EGFR, PIKfyve, we hypothesize that these deregulated miRNAs exhibit coordinated up-regulation of EGFR/PI3K/Akt signaling, thereby contributing to fetal overgrowth in GDM patients during pregnancy.

Among the miRNAs identified, we were drawn to miR-508-3p because it is the most up-regulated miRNA and predicted to target PIKfyve. PIKfyve is a FYVE-containing phosphoinositide 3-phosphate (PI3P) 5 kinase which phosphorylates phosphatidylinositol (PtdIns) 3P to PtdIns $(3,5)$ P2. PIKfyve operates in diverse signaling pathways and membrane trafficking events [17]. Most recently, PIKfyve has been characterized as a negative regulator of EGFR. Er,EE et al have shown that upon EGF stimulation, Akt phosphorylates and activates the kinase PIKfyve, which promotes vesicle trafficking to lysosomes. PIKfyve activation promotes EGFR degradation, suggesting that activation of PIKfyve is to be a feedback mechanism for terminating EGFR signaling and reducing receptor abundance [13]. Here, we identified that miR-508-3p was significantly up-regulated in GDM samples and miR508-3p directly targets PIKfyve. These results indicate that upregulation of miR-508-3p suppress PIKfyve and prevents PIKfyve-mediated EGFR degradation, thereby contributing to constitutive activation of EGFR signaling in GDM patients.

Eight miRNAs including miR-27a, miR-9, miR-137, miR-92a, miR-33a, miR-30d, miR362-5p and miR-502-5p were predicted to identify target genes involved in EGFR/PI3K/Akt pathway and to be down-regulated in GDM tissues. Therefore, there would be a coordinated up-regulation of EGFR/PI3K/Akt pathway-related genes and this was also confirmed in our study. MiR-27a has been shown to exhibit coordinated regulation of the EGFR pathway in 


\section{Cellular Physiology Cell Physiol Biochem 2015;37:243-252 \begin{tabular}{l|l|l} 
DOI: 10.1159/000430349 & O 2015 S. Karger AG, Basel \\
\hline
\end{tabular} \begin{tabular}{l|l} 
and BiOChemistry Published online: August 20, 2015 & www.karger.com/cpb
\end{tabular} \\ Li et al.: A MicroRNA Signature in Gestational Diabetes Mellitus}

solid tumors [18] and also suppress EV71 replication by directly targeting EGFR [19]. MiR137 was found to regulate gastric carcinogenesis by targeting PI3K/Akt pathway [20]. MiR92a and miR-33a were identified to target PI3K/Akt pathway [21, 22]. Further studies are required to understand the roles of each miRNA identified here in GDM.

Taken together, our data identify a miRNA signature involvement in GDM through targeting EGFR/PI3K/Akt pathway and provide new potential insights into the mechanisms of GDM associated with risk of macrosomia.

\section{Acknowledgments}

This work was supported by grants from Beijing Natural Science Foundation (No.7122109), National Natural Science Foundation of China (No. 81472252, No. 31400151), and National Major Scientific and Technological Special Project for "Major New Drugs Innovation and Development" (2014ZX09101041). We are grateful to Mrs. Lanqing Ma (Institute of Microbiology, Chinese Academy of Sciences) for her technical assistance.

\section{Disclosure Statement}

The authors declare that they have no conflict of interest.

\section{References}

1 Grissa O, Yessoufou A, Mrisak I, Hichami A, Amoussou-Guenou D, Grissa A, Djrolo F, Moutairou K, Miled A, Khairi H, Zaouali M, Bougmiza I, Zbidi A, Tabka Z, Khan NA: Growth factor concentrations and their placental mrna expression are modulated in gestational diabetes mellitus: Possible interactions with macrosomia. BMC Pregnancy Childbirth 2010;10:7.

2 Forbes K, Westwood M: Maternal growth factor regulation of human placental development and fetal growth. J Endocrinol 2010;207:1-16.

3 Kristensen P, Susser E, Irgens LM, Mehlum IS, Corbett K, Bjerkedal T: The association of high birth weight with intelligence in young adulthood: A cohort study of male siblings. Am J Epidemiol 2014;180:876-884.

4 Fondacci C, Alsat E, Gabriel R, Blot P, Nessmann C, Evain-Brion D: Alterations of human placental epidermal growth factor receptor in intrauterine growth retardation. J Clin Invest 1994;93:1149-1155.

5 Miettinen PJ, Berger JE, Meneses J, Phung Y, Pedersen RA, Werb Z, Derynck R: Epithelial immaturity and multiorgan failure in mice lacking epidermal growth factor receptor. Nature 1995;376:337-341.

6 Moll SJ, Jones CJ, Crocker IP, Baker PN, Heazell AE: Epidermal growth factor rescues trophoblast apoptosis induced by reactive oxygen species. Apoptosis 2007;12:1611-1622.

7 Liu L, Zhang X, Rong C, Rui C, Ji H, Qian YJ, Jia R, Sun L: Distinct DNA methylomes of human placentas between pre-eclampsia and gestational diabetes mellitus. Cell Physiol Biochem 2014;34:1877-1889.

8 Bartel DP: Micrornas: Target recognition and regulatory functions. Cell 2009;136:215-233.

9 Bartel DP: Micrornas: Genomics, biogenesis, mechanism, and function. Cell 2004;116:281-297.

10 Qing S, Yuan S, Yun C, Hui H, Mao P, Wen F, Ding Y, Liu Q: Serum mirna biomarkers serve as a fingerprint for proliferative diabetic retinopathy. Cell Physiol Biochem 2014;34:1733-1740.

11 Zhao C, Dong J, Jiang T, Shi Z, Yu B, Zhu Y, Chen D, Xu J, Huo R, Dai J, Xia Y, Pan S, Hu Z, Sha J: Early secondtrimester serum mirna profiling predicts gestational diabetes mellitus. PLoS one 2011;6:e23925.

12 Scifres CM, Nelson DM: Intrauterine growth restriction, human placental development and trophoblast cell death. J Physiol 2009;587:3453-3458.

13 Er EE, Mendoza MC, Mackey AM, Rameh LE, Blenis J: Akt facilitates egfr trafficking and degradation by phosphorylating and activating pikfyve. Sci Signal DOI: 10.1126/scisignal.2004015 


\section{Cellular Physiology Cell Physiol Biochem 2015;37:243-252

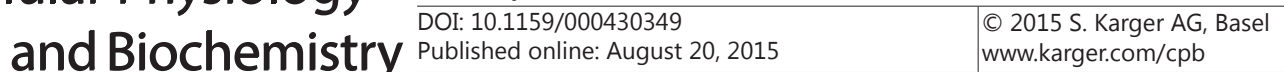

14 Dabelea D, Snell-Bergeon JK, Hartsfield CL, Bischoff KJ, Hamman RF, McDuffie RS: Increasing prevalence of gestational diabetes mellitus ( $\mathrm{gdm}$ ) over time and by birth cohort: Kaiser permanente of colorado gdm screening program. Diabetes Care 2005;28:579-584.

15 Gambino YP, Maymo JL, Perez-Perez A, Duenas JL, Sanchez-Margalet V, Calvo JC, Varone CL: 17betaestradiol enhances leptin expression in human placental cells through genomic and nongenomic actions. Biol Reprod 2010;83:42-51.

16 Hofmann GE, Abramowicz JS: Epidermal growth factor (egf) concentrations in amniotic fluid and maternal urine during pregnancy. Acta Obstet Gynecol Scand1990;69:217-221.

17 Shisheva A: Pikfyve: Partners, significance, debates and paradoxes. Cell Biol Int 2008;32:591-604.

18 Wu X, Bhayani MK, Dodge CT, Nicoloso MS, Chen Y, Yan X, Adachi M, Thomas L, Galer CE, Jiffar T, Pickering CR, Kupferman ME, Myers JN, Calin GA, Lai SY: Coordinated targeting of the egfr signaling axis by microrna27a*. Oncotarget 2013;4:1388-1398.

19 Zhang L, Chen X, Shi Y, Zhou B, Du C, Liu Y, Han S, Yin J, Peng B, He X, Liu W: Mir-27a suppresses ev71 replication by directly targeting egfr. Virus Genes 2014;49:373-382.

20 Cheng Y, Li Y, Liu D, Zhang R, Zhang J: Mir-137 effects on gastric carcinogenesis are mediated by targeting cox-2-activated pi3k/akt signaling pathway. FEBS Lett 2014;588:3274-3281.

21 Ke TW, Wei PL, Yeh KT, Chen WT, Cheng YW: Mir-92a promotes cell metastasis of colorectal cancer through pten-mediated pi3k/akt pathway. Ann Surg Oncol DOI: 10.1245/s10434-014-4305-2.

22 Li ZJ, Ou-Yang PH, Han XP: Profibrotic effect of mir-33a with akt activation in hepatic stellate cells. Cell Signal 2014;26:141-148. 